\title{
OCT in the Management of Diabetic Macular Edema
}

\author{
Nadia K. Waheed · Jay S. Duker
}

Published online: 24 July 2013

(c) Springer Science + Business Media New York 2013

\begin{abstract}
Diabetic macular edema (DME) is the most common cause of mild-to-moderate visual loss in diabetes. With the introduction of anti-vascular endothelial growth factor therapies in addition to the previously available medical and laser therapies, OCT has become the cornerstone in the diagnosis, monitoring, therapeutic selection, and gauging response to therapy in eyes with DME. A review of the recent literature shows numerous advancements in the way OCT scanning is used both to monitor DME and to guide the management of DME.
\end{abstract}

\section{Introduction}

Diabetic retinopathy is estimated to affect one-third of patients with diabetes. The prevalence and severity are affected by the duration of diabetes, glycemic control, and the presence of concurrent hypertension. Epidemiologic studies suggest that diabetic macular edema (DME) will affect up to $7 \%$ of patients with diabetes [1]. DME is the most common cause of mild-to-moderate visual loss in diabetics, and studies confirm that its presence leads to a significantly diminished quality of life and disability [2]. This impact is especially significant because many of the patients suffering vision loss in diabetes are in the working age group and the presence of macular edema results in significant productivity impact on the individual as well as that of society at large.

The Early Treatment Diabetic Retinopathy study defined the term 'clinically significant macular edema' (CSME) that represented the minimal level of edema based on clinical

N. K. Waheed · J. S. Duker $(\bowtie)$

New England Eye Center, Tufts Medical Center, Tufts

University School of Medicine, Boston, MA, USA

e-mail: jduker@tuftsmedicalcenter.org examination that warranted treatment with laser photocoagulation. CSME is thickening of the retina at or within $500 \mu \mathrm{m}$ of the center of the macula, hard exudates at or within $500 \mu \mathrm{m}$ of the center of the macula if associated with thickening of adjacent retina, or a zone of retinal thickening 1 disc area or larger any part of which is within 1 disc diameter of the center of the macula [3]. While this definition is still valid for eyes that have DME not involving the center of fovea, in the era of OCT-guided anti-vascular endothelial growth factor (VEGF) therapy, the decision to treat DME also includes 'center involving' DME, determined by OCT central subfield thickness of greater than or equal to $275 \mu \mathrm{m}$ [4].

While a careful biomicroscopic exam remains essential in the diagnosis and management of DME, OCT scanning provides invaluable information such as quantification of retinal thickness, mapping of the area of thickening, location of fluid (intraretinal or subretinal), the presence of other abnormalities such as vitreomacular traction and epiretinal membrane formation, and the evaluation of foveal microstructure and retinal layers that may be an indicator of the degree of potential visual recovery after treatment of the macular edema (Fig. 1).

\section{Quantitative Measures on OCT Scanning}

Commercial OCT machines provide several quantitative and automated measures that are useful in the evaluation and longitudinal monitoring of patients with DME. These include retinal thickness and the average (mean) central subfield thickness (CSMT or CST). Although the center point retinal thickness can also be measured manually, there is considerable inter-observer variability depending on where the center point is assumed to be on scanning. Thus, central retinal thickness measurement in diabetics has been mostly replaced 
Fig. 1 DME with epiretinal membrane (arrow), cystic changes (arrowhead) and subretinal fluid (large arrow)

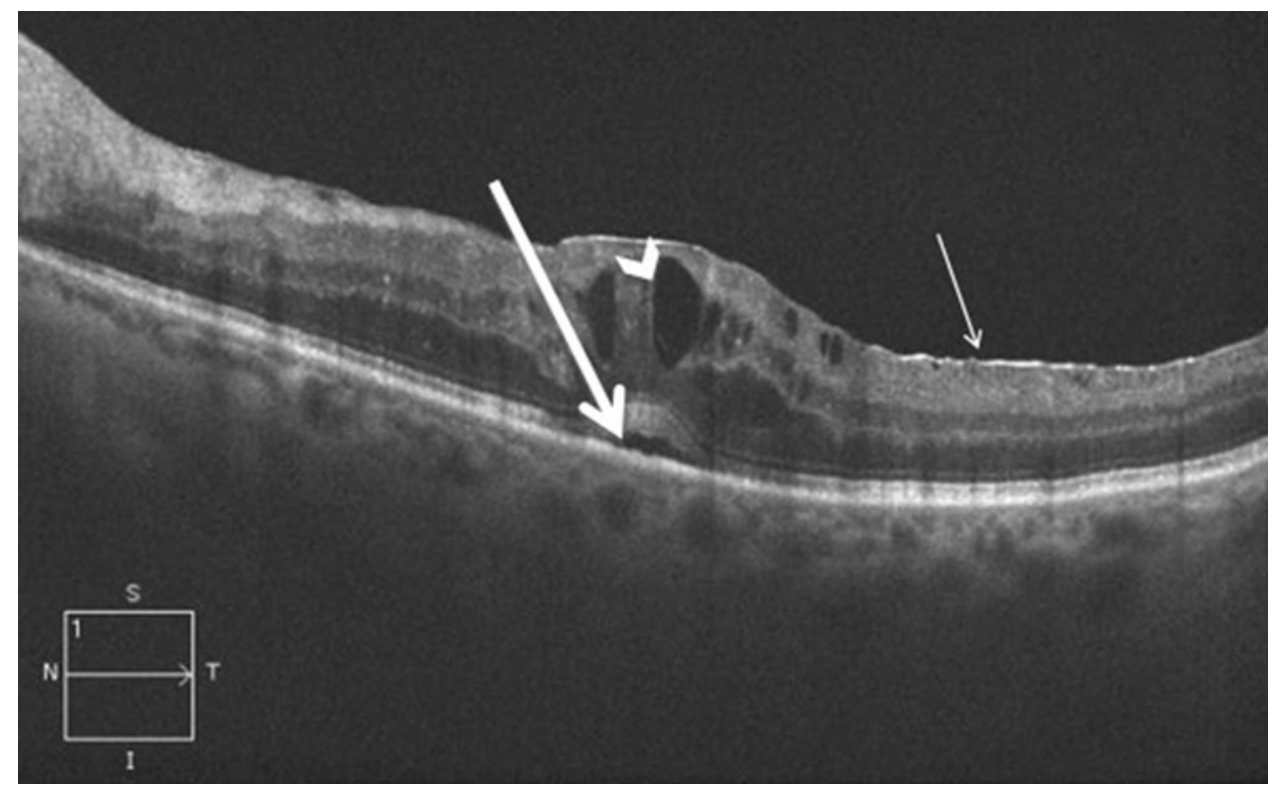

by the automated mean central subfield thickness measurement, which has been shown to be closely correlated to the center point thickness in diabetic patients $[\bullet 5, \bullet \bullet, 7]$.

The mean central subfield thickness is often used as the critical measure in the diagnosis and management of patients with DME. This is defined as the mean retinal thickness within a $1-\mathrm{mm}$ circle centered on the fovea. As previously mentioned, a CST of greater than or equal to $275 \mu \mathrm{m}$ was used as a threshold to define "center-involving DME". However, this value remains under debate. Most large clinical trials including pharmaceutical sponsored trials, and those conducted by the DRCR.net traditionally utilized time domain OCT (TD-OCT) scanning. Time domain OCT is now largely supplanted by spectral domain (SD) OCT in most clinical practices. Although the pathology seen is comparable between the TD and SDOCT scans, SD OCT provides better resolution and more rapid scan acquisition. In addition, it is important to understand that there are measurement differences between the SD- and TD-OCT devices. Especially pertinent to DME, retinal thickness is measured differently between TD- and SD-OCT devices and the numbers obtained are not comparable across systems, neither are they comparable between different SD-OCT systems. This is primarily because of the different segmentation algorithms used in the commercial OCT devices, which measure thickness from different points around the RPE layer [•5]. The Cirrus SD-OCT (Carl Zeiss Meditec), for example, reports significantly greater retinal thicknesses than the Stratus TDOCT (Carl Zeiss Meditec) for the same patient imaged within a few minutes on the two machines. There have been recent attempts to derive 'conversion factors' that would allow for the conversion of retinal thickness obtained from one machine to another, but these are not as yet well established enough to have gained widespread traction [8]. Suffice to say that a value of $275 \mu \mathrm{m}$ as a cutoff for treatment is too low for any SD OCT machine.

Whereas previous thresholds defined on the TD-OCT typically accounted for a $10 \%$ margin of error, there is now some evidence that measurements on the newer SD-OCT may have better reproducibility. A recent study by Comyn et al. looking at the repeatability and reproducibility of OCT measures of retinal thickness in eyes with DME has found that, on the Spectralis SD-OCT device (Heidelberg), changes in central subfield thickness $>8 \mu \mathrm{m}$ can be considered more indicative of true clinical change rather than measurement variability [9]. However, since the study utilized only the Spectralis, it is not clear whether the margin of error on other SD-OCT devices are comparable since the Spectralis uses eye tracking. Moreover, the study did not simulate a real clinical scenario with different operators, but was conducted by the same experienced operator. Other studies demonstrate up to a $17 \%$ diurnal variation in retinal thickness in diabetics, with the least OCT-measured retinal thickness in the late afternoon and the greatest thickness in the early morning [10].

The retinal thickness map obtained on volumetric scanning is a useful adjunct in clinical practice, providing a map of areas of retinal thickness, as well as highlighting areas of thickening outside the central subfield but that may still be classified as CSME. An important limitation, however, of the automated quantitative OCT scans is segmentation breakdown in which the inner or outer retinal boundary is incorrectly identified resulting in abnormal retinal thickness measurements. This has been found to occur in up to $37 \%$ of scans of the central $1 \mathrm{~mm}$ in both the Cirrus and the Spectralis OCT machines [11]. Thus, careful evaluation of the OCT data 
Fig. 2 a Diabetic macular edema with intact IS-OS ellipsoid layer (arrow) and ELM (arrowhead). b DME with disruption of the ELM and IS-OS ellipsoid layer

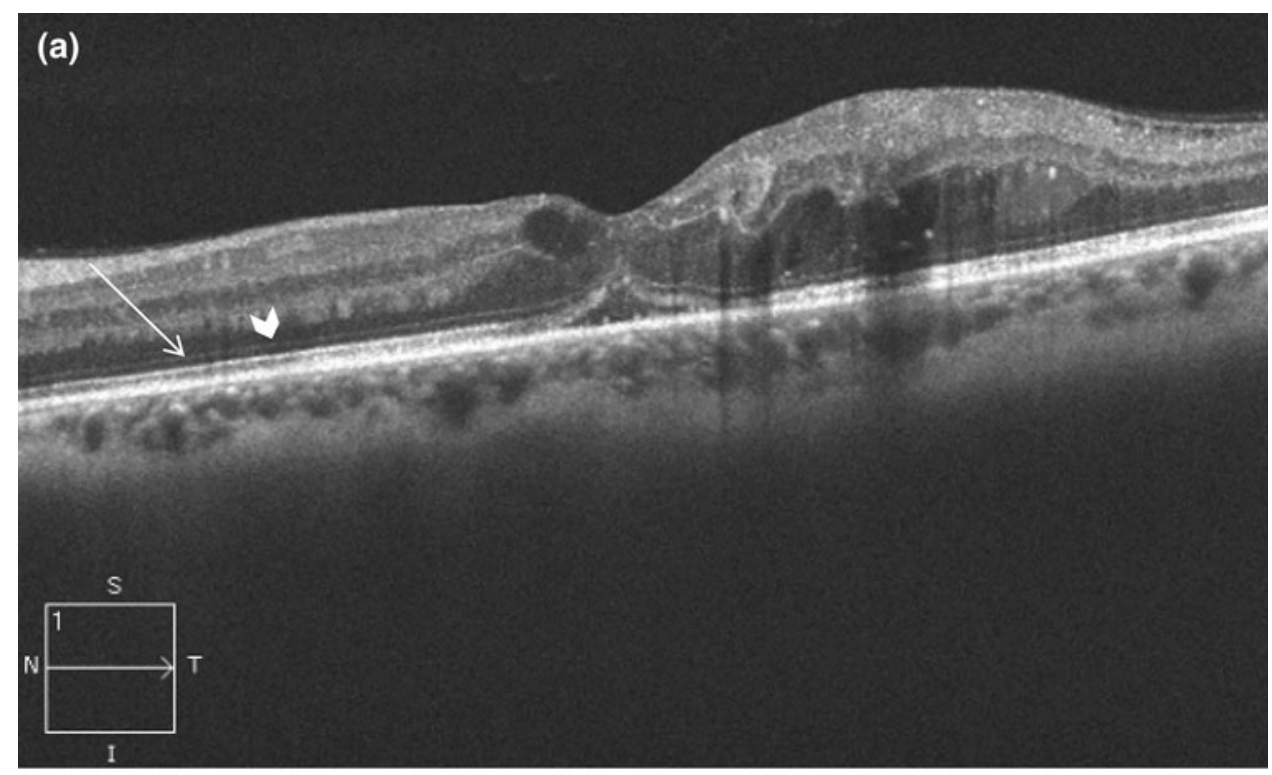

(b)

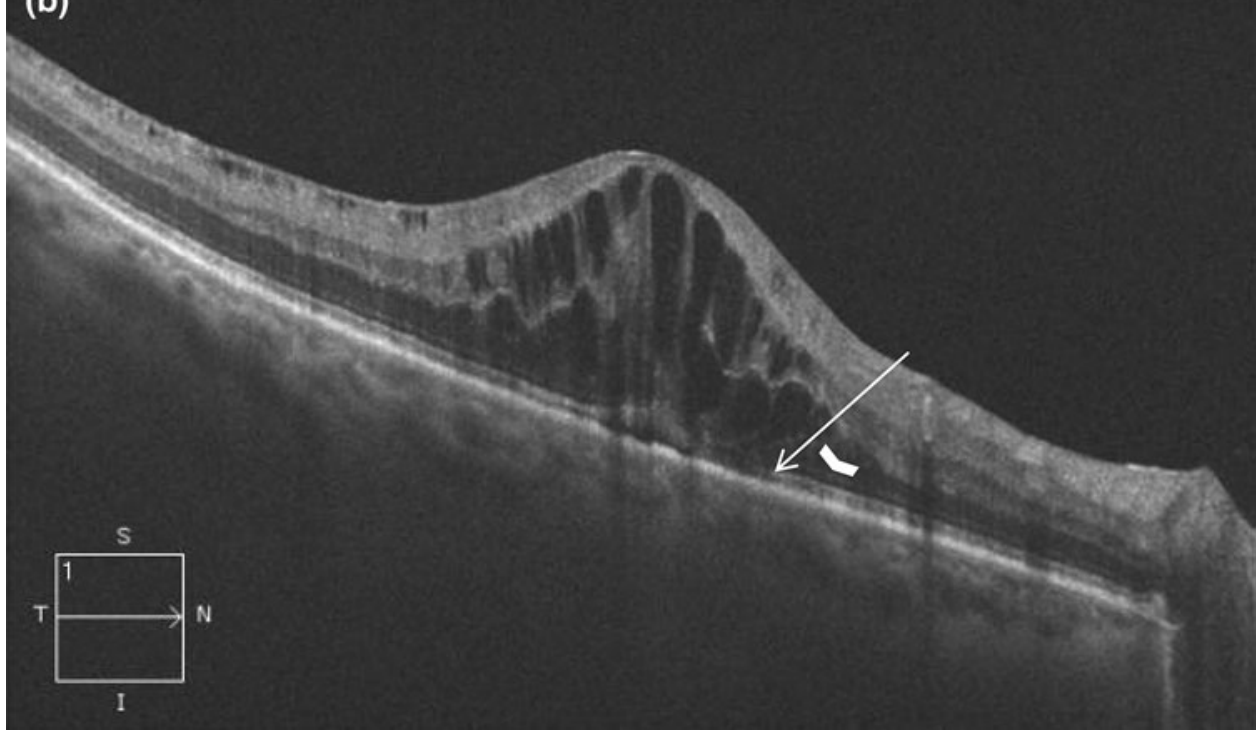

is important in preventing these errors from making diagnostic mistakes in patients with DME. Another limitation of thickness measurements is that they may not necessarily correlate well with visual acuity and visual prognosis. Central subfield thickness has been shown to correlate only modestly with visual acuity in patients with DME [•12]. Thus, subfield thickness by itself is not a good surrogate for post-treatment visual acuity, and qualitative assessment of OCT scans is an important part of the evaluation of eyes with DME.

\section{Qualitative Measures in Diabetic Macular Edema}

The classic clinical definition of DME includes 'focal' and 'diffuse' edema, based on the clinical and angiographic appearance of the DME [3]. The OCT findings of DME are categorized into four major types: thickening of the fovea with homogenous optical reflectivity throughout the whole layer of the retina; thickening of the fovea with markedly decreased optical reflectivity in mostly the outer retinal layers (cystoid changes); thickening of the fovea with subfoveal fluid accumulation and distinct outer border of detached retina; and thickening of the fovea with epiretinal membrane formation with or without apparent vitreofoveal traction [13-15].

The qualitative assessment of OCT scans in DME are proving increasingly important in prognosticating patients as well as determining what patients will respond best to treatment, as our ability to visualize retinal microstructure with the newer OCT machines increases. The presence of homogenous thickening or small cystoid spaces within the outer retina defines patients who usually respond well to 
Fig. 3 Hyper-reflective foci (arrow) and microaneurysm (arrowhead) in diabetic macular edema

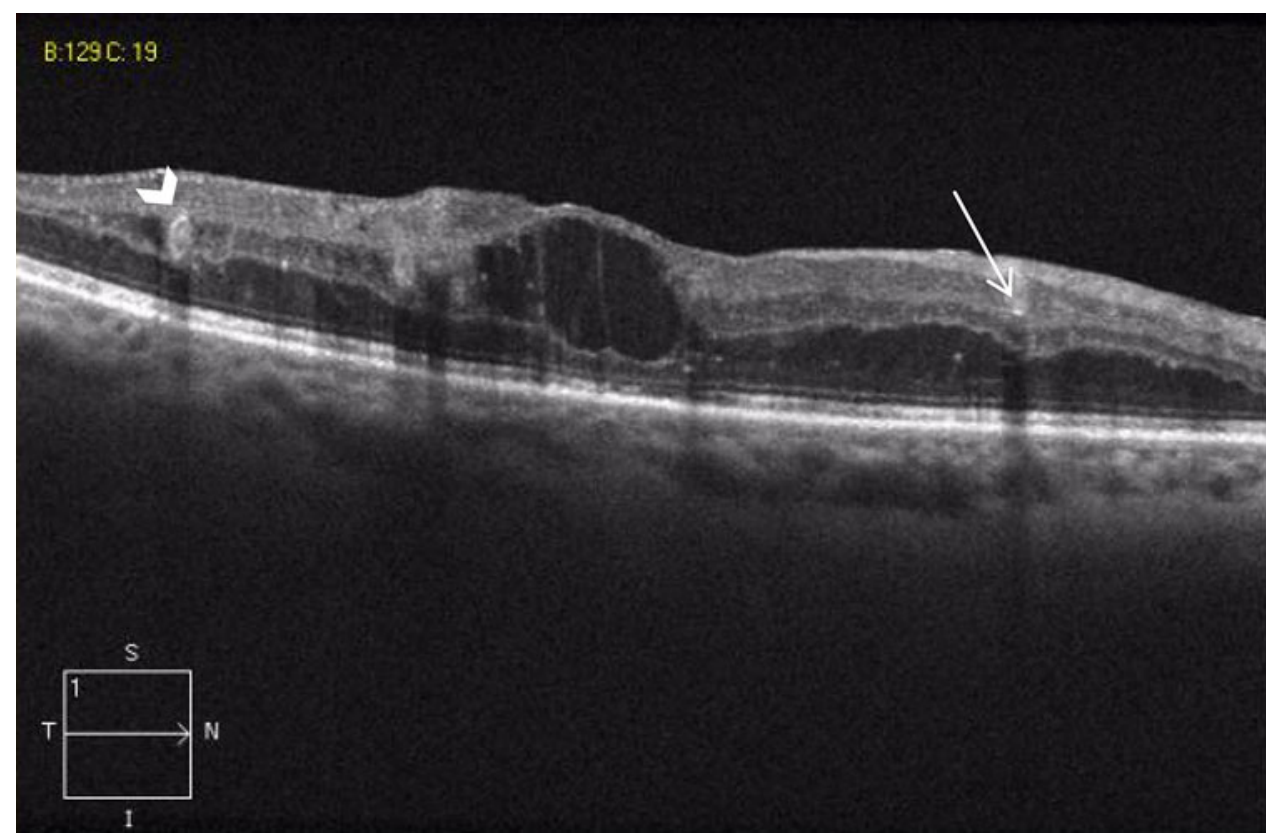

laser and anti-VEGF therapy. The presence of vitreoretinal traction and/or an epiretinal membrane, associated with a loss of the foveal depression and the presence of a taut, thickened posterior hyaloid, has been associated with edema that may be refractory to medical therapy and may respond better to a pars plana vitrectomy with peeling of the posterior hyaloid [11].

Moreover, OCT scanning has also been used to look at foveal microstructural changes such as disruption of the IS-OS ellipsoid layer and external limiting membrane (ELM) (Fig. 2). There is increasing data that indicates that disruption of the IS/OS ellipsoid layer correlates with visual acuity in DME and may be a better predictor of final post-treatment visual acuity than macular thickness [16]. Yohannan et al. $[\bullet 17]$, in a study that correlated retinal point sensitivity by microperimetry with IS-OS ellipsoid disruption on OCT scans, demonstrated a reduction in point sensitivity in eyes with DME that had a disruption in the IS-OS ellipsoid layer. Taking this a step further, Lee et al. [18] tried to correlate macular ischemia on fluorescein angiography in patients with diabetic retinopathy to OCT parameters and found that ischemia on fluorescein angiography is related to the length of the photoreceptor outer segment on SD-OCT scanning and that IS-OS ellipsoid disruption is correlated with visual acuity in ischemic DME. Although further studies are needed to corroborate these results, the length of the outer segment of photoreceptors may be a surrogate for fluorescein angiography in identifying macular ischemia and pre-treatment evaluation of the IS-OS junction may be an important prognosticating factor in the treatment of DME.
Evaluation of ELM disruption has also been studied extensively in patients with DME. There is some controversy about whether loss of the ELM line on OCT represents a true alteration in the microstructure of the outer retina or is an artifact of scanning resulting from an alteration in the orientation of the photoreceptors to incident light in the setting of extracellular fluid. Recent studies suggest that evaluation of ELM preoperatively may predict visual improvement more accurately than the IS-OS ellipsoid layer continuity and central macular thickness. However, this study was performed in a subgroup of eyes with DME undergoing vitrectomy because the macular edema was refractory to medical treatment [19]. Some strides have also been made in the automated quantification of the extent of ELM disruption in patients with DME [19, 20, 21]. While the jury is still out on whether ELM disruption on OCT is a true microstructural alteration in the outer retina, its presence on the OCT may provide yet another prognosticating factor in patients with DME.

Another OCT parameter that has recently garnered significant interest has been the presence of hyper-reflective foci within the outer retina on OCT scanning in DME (Fig. 3). These foci have also been shown in wet agerelated macular degeneration to resolve with anti-VEGF treatment. Whereas in AMD, in which the pathology is primarily subretinal and these hyper-reflective foci probably represent lipid deposition, in DME these hyper-reflective foci may represent a larger variety of microstructural pathologies, including microaneurysms and hard exudates. Interestingly, the baseline amount of hyper-reflective foci seems to correlate positively with $\mathrm{HbA1c}$ values indicating 
the severity of disease $[\cdot 22]$. In patients with DME, these foci were noticed to decrease with anti-VEGF therapy with a significant hyper-reflective foci reduction observable mainly in cases of complete edema resolution; however, no distinct correlation with visual acuity was noticed, presumably mainly due to the enhanced inhomogeneity in the disease progression of DME [•22]. Moreover, the presence of hyper-reflective foci in the outer retina is closely associated with a disrupted ELM and IS-OS ellipsoid layer on SD-OCT images and decreased visual acuity in DME [•22].

Microaneurysms are fairly well characterized on OCT scanning $[21, \cdot 23,24,25]$. They appear as hyper-reflective foci, mostly within the outer half of the retina, usually spanning more than one retinal layer. Many are observed to have an inner homogenous lumen with moderate reflectivity surrounded by a hyper-reflective rim [ $\cdot 23]$. Hyporeflectivity around the microaneurysm is usually associated with leakage on fluorescein angiography [•23]. A recently published study suggests that microaneurysm closure can be evaluated by OCT scanning following laser photocoagulation [18]. Microaneurysm closure is associated with resolution of hyper-reflectivity or by a smaller lumen with heterogenous hyper-reflectivity. Another small pilot study suggested that SD-OCT could be considered a valid alternative to fluorescein angiography in the guidance of macular laser photocoagulation treatment for DME [•26, 27].

In clinical practice as well as in studies, OCT is being used on a routine basis in the diagnosis of DME. Moreover, in addition to visual acuity, it is probably the single most important test in the management of macular edema, monitoring the edema on follow up, establishing treatment criteria, prognostication, and as an outcome measure in clinical trials $[9,14,16]$.

\section{Conclusions}

OCT scanning has emerged as the single most important ancillary test in the evaluation and management of DME [16]. SD-OCT, with better resolution, provides important structural information on the status of the retina in DME. Better depth penetration with enhanced depth imaging technology is also providing us with more data on the role of the choroid in diabetic retinopathy and its progression [28]. Moreover, newer OCT technologies, such as the swept-source and ultra-high resolution systems, provide better visualization of the retinal architecture, and much better axial resolution [29]. These systems are being used to evaluate the role of the vitreo-retinal interface in DME, as well as further delineation of outer retinal changes that may guide treatment in the future. C-Scan imaging on OCT scans and Doppler OCT scanning are being investigated for their role in the evaluation of retinal vasculature, and may soon make invasive testing such as fluorescein angiography redundant. This is coupled with software enhancements that will provide us with better and more reliable segmentation in the future, increasing the accuracy and reproducibility of the data we use to manage DME.

Disclosure Nadia K Waheed and Jay S Duker declare that they have no conflict of interest.

Human and Animal Rights and Informed Consent This article does not contain any studies with human or animal subjects performed by any of the authors.

\section{References}

Papers of particular interest, published recently, have been highlighted as:

- Of importance

•- Of major importance

1. Ding J, Wong TY. Current epidemiology of diabetic retinopathy and diabetic macular edema. Curr Diab Rep. 2012;12(4):346-54. doi:10.1007/s11892-012-0283-6.

2. Hariprasad SM, Mieler WF, Grassi M, Green JL, Jager RD, Miller L. Vision-related quality of life in patients with diabetic macular oedema. Br J Ophthalmol. 2008;92(1):89-92.

3. Photocoagulation for diabetic macular edema. Early treatment diabetic retinopathy study report number 1 . Early treatment diabetic retinopathy study research group. Arch Ophthalmol. 1985;103:1796-806.

4. Nguyen QD, Brown DM, Marcus DM, Boyer DS, Patel S, Feiner L, Gibson A, Sy J, Rundle AC, Hopkins JJ, Rubio RG, Ehrlich JS, RISE and RIDE Research Group. Ranibizumab for diabetic macular edema: results from 2 phase III randomized trials: RISE and RIDE. Ophthalmology. 2012;119(4):789-801.

5. - Wolf-Schnurrbusch UE, Ceklic L, Brinkmann CK, Iliev ME, Frey M, Rothenbuehler SP, Enzmann V, Wolf S. Macular thickness measurements in healthy eyes using six different optical coherence tomography instruments. Invest Ophthalmol Vis Sci. 2009;50(7):3432-7. Important study because compares OCT thickness across machines.

6. • Davis MD, Bressler SB, Aiello LP, et al. Comparison of timedomain OCT and fundus photographic assessments of retinal thickening in eyes with diabetic macular edema. Invest Ophthalmol Vis Sci. 2008;49:1745-52. Important because it compares fundus photographic assessment with OCT scanning.

7. Krzystolik MG, Strauber SF, Aiello LP, et al. Reproducibility of macular thickness and volume using Zeiss optical coherence tomography in patients with diabetic macular edema. Ophthalmology. 2007; 114:1520-5.

8. Ibrahim MA, Sepah YJ, Symons RC, Channa R, Hatef E, Khwaja A, Bittencourt M, Heo J, Do DV, Nguyen QD. Spectral- and time-domain optical coherence tomography measurements of macular thickness in normal eyes and in eyes with diabetic macular edema. Eye (Lond). 2012;26(3):454-62.

9. Comyn O, Heng LZ, Ikeji F, Bibi K, Hykin PG, Bainbridge JW, Patel PJ. Repeatability of spectralis OCT measurements of macular thickness and volume in diabetic macular edema. Invest Ophthalmol Vis Sci. 2012;53(12):7754-9. 
10. Diabetic Retinopathy Clinical Research Network, Danis RP, Glassman AR, Aiello LP, Antoszyk AN, Beck RW, Browning DJ, Ciardella AP, Kinyoun JL, Murtha TJ, Topping TM, Shami M, Sharuk GS, Wells JA 3rd. Diurnal variation in retinal thickening measurement by optical coherence tomography in center-involved diabetic macular edema. Arch Ophthalmol. 2006;124(12):1701-7.

11. Han IC, Jaffe GJ. Evaluation of artifacts associated with macular spectral-domain OCT. Ophthalmology. 2010;117:1177-89.

12. - Alasil T, Keane PA, Updike JF, Dustin L, Ouyang Y, Walsh AC, Sadda SR. Relationship between optical coherence tomography retinal parameters and visual acuity in diabetic macular edema. Ophthalmology. 2010;117(12):2379-86. Important paper on structure function correlation.

13. Diabetic Retinopathy Clinical Research Network, Elman MJ, Qin H, Aiello LP, Beck RW, Bressler NM, Ferris FL 3rd, Glassman AR, Maturi RK, Melia M. Randomized trial evaluating ranibizumab plus prompt or deferred laser or triamcinolone plus prompt laser for diabetic macular edema. Ophthalmology. 2010; 117:1064-77.

14. Virgili G, Menchini F, Dimastrogiovanni AF, Rapizzi E, Menchini U, Bandello F, Chiodini RG. Optical coherence tomography versus stereoscopic fundus photography or biomicroscopy for diagnosing diabetic macular edema: a systematic review. Invest Ophthalmol Vis Sci. 2007;48:4963-73.

15. Catier A, Tadayoni R, Paques M, Erginay A, Haouchine B, Gaudric A, Massin P. Characterization of macular edema from various etiologies by optical coherence tomography. Am J Ophthalmol. 2005;140(2):200-6.

16. Murakami T, Nishijima $\mathrm{K}$, Akagi $\mathrm{T}$, Uji A, Horii $\mathrm{T}$, UedaArakawa N, Muraoka Y, Yoshimura N. Optical coherence tomographic reflectivity of photoreceptors beneath cystoid spaces in diabetic macular edema. Invest Ophthalmol Vis Sci. 2012; 53(3):1506-11.

17. - Yohannan J, Bittencourt M, Sepah YJ, Hatef E, Sophie R, Moradi A, Liu H, Ibrahim M, Do DV, Coulantuoni E, Nguyen QD. Association of retinal sensitivity to integrity of photoreceptor inner/outer segment junction in patients with diabetic macular edema. Ophthalmology. 2013;120(6):1254-61. doi:10.1016/ j.ophtha.2012.12.003. Another important paper on structure function correlation.

18. Lee SN, Chhablani J, Chan CK, Wang H, Barteselli G, El-Emam S, Gomez ML, Kozak I, Cheng L, Freeman WR. Characterization of microaneurysm closure after focal laser photocoagulation in diabetic macular edema. Am J Ophthalmol. 2013;155(5):905-12.

19. Chen X, Zhang L, Sohn EH, Lee K, Niemeijer M, Chen J, Sonka $\mathrm{M}$, Abràmoff MD. Quantification of external limiting membrane disruption caused by diabetic macular edema from SD-OCT. Invest Ophthalmol Vis Sci. 2012;53(13):8042-8.
20. Diabetic Retinopathy Clinical Research Network, Bressler NM, Miller KM, Beck RW, Bressler SB, Glassman AR, Kitchens JW, Melia M, Schlossman DK. Observational study of subclinical diabetic macular edema. Eye (Lond). 2012;26(6):833-40. doi: 10.1038/eye.2012.53.

21. Uji A, Murakami T, Nishijima K, Akagi T, Horii T, Arakawa N, Muraoka Y, Ellabban AA, Yoshimura N. Association between hyperreflective foci in the outer retina, status of photoreceptor layer, and visual acuity in diabetic macular edema. Am J Ophthalmol. 2012;153(4):710-717.e1.

22. - Framme C, Schweizer P, Imesch M, Wolf S, Wolf-Schnurrbusch U. Behavior of SD-OCT-detected hyperreflective foci in the retina of anti-VEGF-treated patients with diabetic macular edema. Invest Ophthalmol Vis Sci. 2012;53(9):5814-8. doi: 10.1167/iovs.12-9950. Important recent paper looking at OCT based classification of features of diabetic macular edema.

23. - Wang H, Chhablani J, Freeman WR, Chan CK, Kozak I, Bartsch DU, Cheng L. Characterization of diabetic microaneurysms by simultaneous fluorescein angiography and spectraldomain optical coherence tomography. Am J Ophthalmol. 2012;153(5):861-867.e1. doi:10.1016/j.ajo.2011.10.005. Important paper on OCT based classification of diabetic macular edema.

24. Lee Dong-Hoon, Kim JT, Da-Woon J, Joe SG, Yoon YH. The relationship between foveal ischemia and spectral-domain optical coherence tomography findings in ischemic diabetic macular edema. Invest Ophthalmol Vis Sci. 2013;54(2):1080-5.

25. Chhablani JK, Kim JS, Cheng L, Kozak I, Freeman W. External limiting membrane as a predictor of visual improvement in diabetic macular edema after pars plana vitrectomy. Graefes Arch Clin Exp Ophthalmol. 2012;250(10):1415-20.

26. - Kang SW, Park CY, HAM DI. The correlation between fluorescein angiographic and optical coherence tomographic features in clinically significant diabetic macular edema. Am J Ophthalmol. 2004;137(2):313-22. Important because it is another paper on OCT based classification of the features of diabetic macular edema.

27. Gallego-Pinazo R, Suelves-Cogollos AM, Dolz-Marco R, Arevalo JF, García-Delpech S, Mullor JL, Díaz-Llopis M. Macular laser photocoagulation guided by spectral-domain optical coherence tomography versus fluorescein angiography for diabetic macular edema. Clin Ophthalmol. 2011;5:613-7.

28. Querques G, Lattanzio R, Querques L, Del Turco C, Forte R, Pierro L, Souied EH, Bandello F. Enhanced depth imaging optical coherence tomography in type 2 diabetes. Invest Ophthalmol Vis Sci. 2012;53(10):6017-24.

29. Adhi M, Duker JS. Optical coherence tomography-current and future applications. Curr Opin Ophthalmol. 2013;24(3):213-21. 